\title{
Association of chronic stress, inflammation, body composition and dietary intake in Croatian university students
}

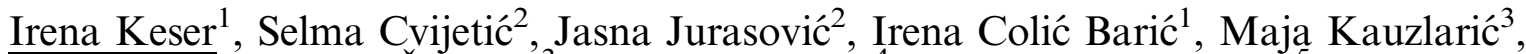 \\ Katarina Simunić ${ }^{3}$, Dario Boschiero and Jasminka Z. Ilich ${ }^{5}$ \\ ${ }^{1}$ Laboratory for Nutrition Science, Faculty of Food Technology and Biotechnology, University of Zagreb, Zagreb, \\ Croatia, \\ ${ }^{2}$ Institute for Medical Research and Occupational Health, Zagreb, Croatia, \\ ${ }^{3}$ Graduate student, Faculty of Food Technology and Biotechnology, University of Zagreb, Zagreb, Croatia, \\ ${ }^{4}$ BioTekna S.r.l., Marcon-Venice, Italy and \\ ${ }^{5}$ Institute for Successful Longevity, Florida State University, Tallahassee, USA
}

\begin{abstract}
Introduction: Chronic stress and low-grade chronic inflammation (LGCI) influence body composition and are key underlying factors in health and disease. New syndrome, osteosarcopenic obesity (OSO), signifies the simultaneous impairment of bone, muscle, and adipose tissues and has been associated with poor diet and metabolic derangements. Hyperactivation of the hypothalamic-pituitary-adrenal (HPA) axis associated with hypercortisolemia and LGCI disrupts the metabolism of bone, muscle and, adipose tissue and may cause changes in eating behavior. Some nutrients involved in preventing/alleviating OSO and LCGI include protein, calcium, magnesium, vitamin D, fiber and omega-3 fatty acids. The aim of this study was to examine the relations between chronic stress, LGCI, body composition and dietary intake in university students.
\end{abstract}

Materials and Methods: Participants were $\mathrm{n}=50$ graduate nutritional students (Age, $23.0 \pm 1.4$ years; $\mathrm{BMI}, 22.1 \pm 2.7 \mathrm{~kg} / \mathrm{m}^{2}$ ) $[\mathrm{n}=45$ females (90\%)]. Advanced bio-impedance devices, BIA-ACC® and PPG-StressFlow ${ }^{\circledR}$ (BioTekna S.r.l., Marcon-Venice, Italy), were used to determine total bone mass $(\mathrm{kg})$ and T-score; muscle mass ( $\mathrm{kg}$ and \% fat free mass) and S-score; and fat mass (kg and \% of body weight), as well as extracellular water and HPA index (reference value > 3.5) as indicators of underlying LGCI and stress. Dietary intake was estimated by 24-hour recall and analyzed for all macro- and micro-nutrients.

Results: Positive correlation was found between HPA index and the intake of either total or animal protein, calcium and riboflavin, with $\mathrm{r}$ ranging from $0.3-0.35$, all $\mathrm{p}<0.05$. HPA index also positively correlated with $\mathrm{T}$-score $(\mathrm{r}=0.39, \mathrm{p}=0.005)$ and S-score $(r=0.49, p=0.0003)$. Total protein intake positively correlated with $\mathrm{T}$-score $(\mathrm{r}=0.32, \mathrm{p}=0.024)$ and animal protein with S-score $(r=0.34, p=0.017)$.

Discussion: Our results show that chronic stress, LGCI and body composition were interrelated in this young population and possibly augmented by higher protein and calcium intake. Screening for body composition dyshomeostasis, chronic stress and LGCI using innovative, non-invasive devices, along with assessing intake of some crucial nutrients, may provide quick and useful health information enabling prevention or early treatment of some linked disorders.

\section{Conflict of Interest}

There is no conflict of interest. 